\title{
IL17: potential therapeutic target in Sjögren's syndrome using adenovirus-mediated gene transfer
}

\author{
Cuong Q Nguyen ${ }^{1,2}$, Hongen Yin ${ }^{3}$, Byung Ha Lee ${ }^{1}$, John A Chiorini ${ }^{3}$ and Ammon B Peck ${ }^{1,2,4}$
}

Sjögren's syndrome (SS) involves a chronic, progressive inflammation primarily of the salivary and lacrimal glands leading to decreased levels of saliva and tears that eventually result in dry mouth and dry eye diseases. $T_{H} 17$ cell populations secreting IL17A have been shown to have an important function in an increasing number of autoimmune diseases, including SS. In this study, we investigated the function of IL17A on SS development and onset. Adenovirus-5 vectors expressing either IL17R:fragment of crystallization $(\mathrm{Fc})$ fusion protein or LacZ were injected through retrograde cannulation into the salivary glands of SS-susceptible $\left(\mathrm{SS}^{\mathrm{S}}\right)$ C57BL/6.NOD-Aec1Aec2 mice between 6 and 8 weeks of age (a pre-disease stage) or 15 and 17 weeks of age (a diseased stage). The mice were subsequently characterized for their SS phenotypes. Mice cannulated with the Ad5-IL17R:Fc viral vector at either 7 or 16 weeks of age exhibited a rapid temporal, yet persistent, decrease in the levels of serum IL17 as well as the overall numbers of CD4 + IL17 + T cells present in their spleens. Disease profiling indicated that these mice showed decreased lymphocytic infiltrations of their salivary glands, normalization of their antinuclear antibodies repertoire, and increased saliva secretion. In contrast, mice cannulated with the control Ad5-LacZ viral vector did not exhibit similar changes and progressed to the overt disease stage. The capacity of the Ad5-IL17R:Fc-blocking factor to reduce SS pathology in SS ${ }^{S}$ mice strongly suggests that IL17 is an important inflammatory cytokine in salivary gland dysfunction. Thus, therapeutic approach targeting IL17 may be effective in preventing glandular dysfunction.

Laboratory Investigation (2011) 91, 54-62; doi:10.1038/labinvest.2010.164; published online 20 September 2010

KEYWORDS: adenoviral vector; gene therapy; IL17; IL17R; Sjögren's syndrome; $T_{H} 17$ cells

Sjögren's syndrome (SS) is a chronic, systemic autoimmune disease characterized most notably by the development of dry eyes and dry mouth manifestations, indicative of secretory dysfunction of the lacrimal and salivary glands. ${ }^{1-3}$ Although an underlying cause of SS remains elusive, studies using the NOD/LtJ and C57BL/6.NOD-Aec1Aec2 mouse models of SS have provided evidence that this autoimmune exocrinopathy progresses through several consecutive, yet distinct, phases. $^{1-3}$ In the first phase, occurring between birth and 6-8 weeks of age, a series of aberrant genetic, physiological, and biochemical activities associated with retarded salivary gland organogenesis and acinar cell apoptosis occur before initiation of detectable autoimmunity. In the second phase, occurring between 8 and 18-20 weeks of age, various leukocyte populations first by antigen-presenting cells, especially dendritic cells followed by $\mathrm{T}$ and $\mathrm{B}$ lymphocytes, infiltrate the exocrine glands with a concomitant increase in the expression of inflammatory cytokines and production of autoantibodies. In the last phase, occurring usually after 18 weeks of age, significant secretory dysfunction of the salivary and lacrimal glands occurs, most likely the result of production of pathogenic autoantibodies reactive against the muscarinic receptor type III. ${ }^{4,5}$ Previous studies have shown that intervention or disruption of the biological or immunological elements identified in one or more of the three phases delays or prevents the subsequent onset of SS in these murine models. ${ }^{5-7}$

Although these earlier studies have implicated both $\mathrm{T}_{\mathrm{H}} 1$ and $\mathrm{T}_{\mathrm{H}} 2$ cell-associated functions in the development and onset of clinical SS, recent identification of the CD4 $+\mathrm{T}_{\mathrm{H}} 17$ memory cells within the lymphocytic focus (LF) of lacrimal and salivary glands of SS-susceptible $\left(\mathrm{SS}^{\mathrm{s}}\right) \mathrm{C} 57 \mathrm{BL} / 6 . \mathrm{NOD}$ Aec1Aec2 mice, as well as minor salivary glands of human SS patients, greatly expands the potential complexity in

\footnotetext{
${ }^{1}$ Department of Oral Biology, University of Florida College of Dentistry, Gainesville, FL, USA; ${ }^{2}$ Center for Orphan Autoimmune Disorders, University of Florida College of Dentistry, Gainesville, FL, USA; ${ }^{3}$ Molecular Physiology and Therapeutics Branch, National Institute of Dental and Craniofacial Research, NIH, Bethesda, MD, USA and ${ }^{4}$ Department of Pathology, Immunology and Laboratory Medicine, University of Florida College of Medicine, Gainesville, FL, USA Correspondence: Dr CQ Nguyen, PhD, Department of Oral Biology, PO Box 100424, College of Dentistry, University of Florida, Gainesville, FL 32610 USA. E-mail: Nguyen@pathology.ufl.edu
} 
deciphering the autoimmune response underlying SS. ${ }^{8,9}$ The $\mathrm{T}_{\mathrm{H}} 17$ cell population, while clearly a subset of $\mathrm{CD} 4+$ memory effector $T$ cells, is distinct from either the $T_{H} 1$ or $\mathrm{T}_{\mathrm{H}} 2$ cell lineages. ${ }^{10-14} \mathrm{~T}_{\mathrm{H}} 17$ effector cells secrete at least one of the six cytokines belonging to the IL17 family, that is IL17A, IL17B, IL17C, IL17D, IL25, and/or IL17F; however, IL17A (IL17), the signature cytokine, has received the greatest attention in studies of autoimmune diseases. ${ }^{15}$ The IL17 cytokines are potent pro-inflammatory molecules, actively involved in tissue inflammation through induction of pro-inflammatory cytokine and chemokine expressions. ${ }^{16}$ In addition, IL17 is involved in the mobilization, maturation, and migration of neutrophils through the release of IL8 at the site of injury. ${ }^{17}$ Interestingly, IL17A is known to regulate Foxp3 $+\mathrm{T}_{\mathrm{Reg}}$ cells and vice versa. ${ }^{18}$

Although $\mathrm{T}_{\mathrm{H}} 17$ cells have been implicated in several autoimmune diseases (eg Crohn's disease, ${ }^{19,20}$ experimental autoimmune encephalomyelitis, ${ }^{21}$ collagen-induced arthritis CIA), ${ }^{21} \mathrm{SS},{ }^{8}$ and others, ${ }^{2,3}$ and this characteristic may require signaling from $\mathrm{T}_{\mathrm{H}} 1$ cells already present in the lesion. ${ }^{3}$ In any event, recent observational studies in SS patients and animal models of primary SS have identified the presence of IL17 and its activating cytokine IL23 in the lymphocytic infiltrates of the exocrine glands, as well as higher levels of circulating IL17 in both sera and saliva, ${ }^{8,9}$ raising the question of the importance of IL17 in SS. Thus, the goals of this study were to determine whether blocking IL17 can directly interfere with the onset of SS-like disease by improving the pathology and clinical signs in the salivary glands and, in part, identify IL17 as a potential therapeutic target in preventing the development or reversing progression of SS-like disease.

\section{MATERIALS AND METHODS Animals}

$\mathrm{SS}^{\mathrm{S}} \mathrm{C}$ 77BL/6.NOD-Aec1Aec2 mice were bred and maintained under specific pathogen-free conditions. The animals were maintained on a $12 \mathrm{~h}$ light-dark schedule and provided food and acidified water ad libitum. At times indicated in the text, mice were euthanized by cervical dislocation after deep anesthetization with isoflurane, after which organs were freshly explanted for analyses. Both the breeding and use of these animals for the present studies were approved by the University of Florida's IACUC. Salivary glands of C57BL/6.NODAec1Aec2 mice were cannulated with IL17-blocking vector, Ad5-IL17R:fragment of crystallization $(\mathrm{Fc})$, using retrograde injections at either 8 weeks of age $(n=9)$ or 17 weeks of age $(n=12)$. Age- and sex-matched control mice $(n=10$ per age group) received the Ad5-LacZ control vector using the same protocol.

\section{Production of Ad5-LacZ and Ad5-IL17R:Fc Vectors}

The recombinant adenovirus vectors used in this study were generously provided by Dr Jay K Kolls (Children's Hospital of Pittsburgh, Pittsburgh, PA, USA). These vectors were constructed based on the first generation serotype 5 adenovirus
(Ad5) and shown to produce their appropriate and functional products. ${ }^{22-24}$ In brief, the Ad5-IL17R:Fc vector was initially made by fusing the extracellular domain of mouse IL17R with the murine IgG1 $\mathrm{CH} 2$ and $\mathrm{CH} 3$ domains. The functionality of the fusion protein was tested by inhibiting recombinant IL17-induced production of IL6 in 3T3 fibroblasts. ${ }^{25}$ The construct was expressed in vivo by transferring the fusion gene into an E1-deleted recombinant adenovirus (Ad5-IL17R:Fc). ${ }^{25}$ To obtain sufficient viral vectors for the present studies, each recombinant vector was amplified in HEK293 cells, purified by two rounds of $\mathrm{CsCl}$ gradient centrifugation, then dialyzed against $100 \mathrm{mM}$ Tris- $\mathrm{HCl}(\mathrm{pH}$ 7.4), $10 \mathrm{mM} \mathrm{MgCl}_{2}$, and $10 \%(\mathrm{v} / \mathrm{v})$ glycerol, as described elsewhere. $^{26}$

\section{Retrograde Salivary Gland Cannulation of Ad5-LacZ or Ad5-IL17R:Fc Vectors}

Previous studies have shown that retrograde salivary gland cannulation is an effective method to direct local gene expression in the salivary glands. ${ }^{27-29}$ In brief, before cannulation, each mouse was anesthetized with a ketamine:xylazine mixture $((100 \mathrm{mg} / \mathrm{ml}, 1 \mathrm{ml} / \mathrm{kg}$ body weight; Fort Dodge Animal Health, Fort Dodge, IA) and xylazine $(20 \mathrm{mg} / \mathrm{ml}$, $0.7 \mathrm{ml} / \mathrm{kg}$ body weight; Phoenix Scientific, St Joseph, MO, USA)) intramuscularly. Stretched PE-10 polyethylene tubes were inserted into each of the two openings of the salivary ducts. After securing the cannulas, the mouse received an intramuscular injection of atropine $(1 \mathrm{mg} / \mathrm{kg})$, followed 10 min later by a slow, steady injection of viral vector. Each salivary gland received $50 \mu \mathrm{l}$ of vector solution containing $10^{7}$ viral particles. This vector dose was chosen based on published literature in which dosage optimizations were performed extensively. ${ }^{30,31}$ The cannulas were removed $5 \mathrm{~min}$ later to ensure successful cannulation.

\section{Measurement of Stimulated Saliva Flow}

To measure stimulated saliva flow, individual non-anesthetized mice were weighed and given an intraperitoneal injection of $100 \mu \mathrm{l}$ of phosphate-buffered saline (PBS) containing isoproterenol $(0.02 \mathrm{mg} / \mathrm{ml})$ and pilocarpine $(0.05 \mathrm{mg} / \mathrm{ml})$ (Sigma-Alrich, St Louis, MO, USA). Saliva was collected for $10 \mathrm{~min}$ from the oral cavity of individual mice using a micropipette starting $1 \mathrm{~min}$ after injection of the secretagogue. The volume of each saliva sample was measured. Before vector cannulation and again at each time point designated in the text, saliva and sera were collected from each mouse. Samples were stored at $-80^{\circ} \mathrm{C}$ until analyzed.

\section{Determination of IL17 Cytokines Level}

Measurement of IL17 in serum samples were performed using the mouse IL17 Bio-Plex Cytokine Assay (Bio-Rad, Hercules, CA, USA). All procedures were performed according to the manufacturer's instructions. Readings were carried out using the Luminex 200 system (Luminex, Austin, TX, USA). Standard curves were generated from 3.1 to 
$10200 \mathrm{pg} / \mathrm{ml}$. The lower cutoff level for detection by the software was $1 \mathrm{pg} / \mathrm{ml}$.

\section{Intracellular Cytokine Staining and Flow Cytometric Analysis}

Splenocytes were prepared as previously described. ${ }^{6}$ Cells were plated in a 24-well microtiter plate pre-coated with antiCD3 $(10 \mu \mathrm{g} / \mathrm{ml})$ and anti-CD28 antibodies $(2 \mu \mathrm{g} / \mathrm{ml})$ and incubated for $5 \mathrm{~h}$ with leukocyte activation cocktail containing GolgiPlug $(2 \mu \mathrm{l} / \mathrm{ml})$. Collected cells were fixed and permeabilized using Cytofix/CytopermFixation/Permeabilization. Flow cytometric acquisition for intracellular staining was performed after staining with PE-Cy5-anti-mouse CD4 and PE-anti-IL17A. The cells were counted on an FACSCalibur (BD, Franklin Lakes, NJ, USA) and analyzed by FCS Express (De Novo Software, Los Angeles, CA, USA).

\section{Histology}

Following euthanasia, salivary glands were surgically removed from each mouse and placed in 10\% phosphatebuffered formalin for $24 \mathrm{~h}$. Fixed tissues were embedded in paraffin and sectioned at $5 \mu \mathrm{m}$ thickness. Three nonconsecutive sections separated by $100 \mu \mathrm{m}$ and cut across the entire glands were used. Paraffin-embedded sections were deparaffinized by immersing in xylene, followed by dehydration in ethanol. The paraffin-embedded salivary glands were prepared and stained with hematoxylin and eosin (H\&E) dye. Stained sections were observed under a microscope for glandular structure and leukocyte infiltration determination. A double-blinded procedure was used to enumerate leukocytic infiltrations in the histological sections of salivary glands. In this study, LF were defined as aggregates of $>50$ leukocytes quantified per each histological section. Calculations were based on a particular histological section with the most severe LF in the gland.

\section{Immunofluorescent Staining for B and T Cells}

Histological sections of salivary glands were incubated with rat anti-mouse B220 (BD Pharmingen, San Jose, CA, USA) and goat anti-mouse CD3 (Santa Cruz Biotechnology, Santa Cruz, CA, USA), followed by incubation with Texas Redconjugated rabbit anti-rat IgG (Biomeda, Foster City, CA, USA) and FITC-conjugated rabbit anti-goat IgG (SigmaAldrich). The slides were mounted with DAPI-mounting medium (Vector Laboratories, Burlingame, CA, USA).

Sections were observed at $\times 200$ magnification using a Zeiss Axiovert 200M microscope and images were obtained with AxioVs40 software (Ver. 4.7.1.0, Zeiss) (Carl Zeiss, Thornwood, NY, USA).

\section{Immunohistochemical Staining for IL17 in Salivary Glands}

Immunohistochemical staining for IL17A was carried out as previously described. ${ }^{8}$ In brief, paraffin-embedded salivary glands were de-paraffinized by immersion in xylene, followed by antigen retrieval with $10 \mathrm{mM}$ citrate buffer, $\mathrm{pH}$ 6.0. Tissue sections were incubated overnight at $4^{\circ} \mathrm{C}$ with anti-IL17 antibody (Santa Cruz Biotechnology). Isotype controls were performed with rabbit IgG. The slides were incubated with biotinylated goat anti-rabbit IgG followed by horseradish peroxidase-conjugated avidin-biotin-peroxidase incubation using the Vectastain $\mathrm{ABC}$ kit. The staining was developed by using diaminobenzidine substrate (Vector Laboratories), and counterstaining was performed with hematoxylin. Stained sections were observed at $\times 200$ magnification using a Zeiss Axiovert 200M microscope and images were obtained with AxioVs40 software (Ver. 4.7.1.0, Zeiss) (Carl Zeiss).

\section{Detection of Antinuclear Antibodies in the Sera}

Antinuclear antibodies (ANA) in the sera of mice were detected using HEp-2 ANA kit (INOVA Diagnostics, San Diego, CA, USA). All procedures were performed according to the manufacturer's instructions. In brief, HEp-2-fixed substrate slides were overlaid with appropriate mouse sera diluted 1:40, $1: 80$, and 1:160. Slides were incubated for $1 \mathrm{~h}$ at room temperature in a humidified chamber. After three washes for 5 min with PBS, the substrate slides were covered with Alexa 488-conjugated goat anti-mouse IgG (H/L) (Invitrogen, Carlsbad, CA, USA) diluted 1:100 for $45 \mathrm{~min}$ at room temperature. After three washes, fluorescence was detected by fluorescence microscopy at $\times 200$ magnification using a Zeiss Axiovert $200 \mathrm{M}$ microscope and all images were obtained with AxioVs40 software with constant exposure of $0.3 \mathrm{~s}$ (Carl Zeiss). In this study, data are the results using 1:40 dilutions of sera from each experimental group.

\section{Statistical Analyses}

Statistical evaluations between saliva collections were determined by using Mann-Whitney $U$-test generated by the GraphPad InStat software (GraphPad Software, La Jolla, CA, USA). The two-tailed $P$-value $<0.05$ was considered significant.

\section{RESULTS \\ Reduction of Serum IL17 Cytokine Levels After Transduction with Ad5-IL17R:Fc Vector}

Although adenoviral vectors have been shown to elicit optimal recombinant gene expressions around day 5 post-infection, which then persist for approximately 2 weeks, ${ }^{32}$ this study used immunohistochemistry staining against LacZ protein to show that optimal transduction efficiency was approximately $26 \pm 5 \%$ at 2 weeks post-infusion, which decreased to $15 \pm 3 \%$ by 9 weeks (data not shown). To determine the efficacy of the Ad5-IL17R:Fc-blocking vector to reduce the systemic levels of IL17 in C57BL/6.NOD-Aec1Aec2 mice after transduction of the salivary glands, IL17 levels were quantified in sera collected at several time points postcannulation.

As shown in Figure 1a, C57BL/6.NOD-Aec1Aec2 mice treated with the Ad5-IL17R:Fc vector at 8 weeks of age 

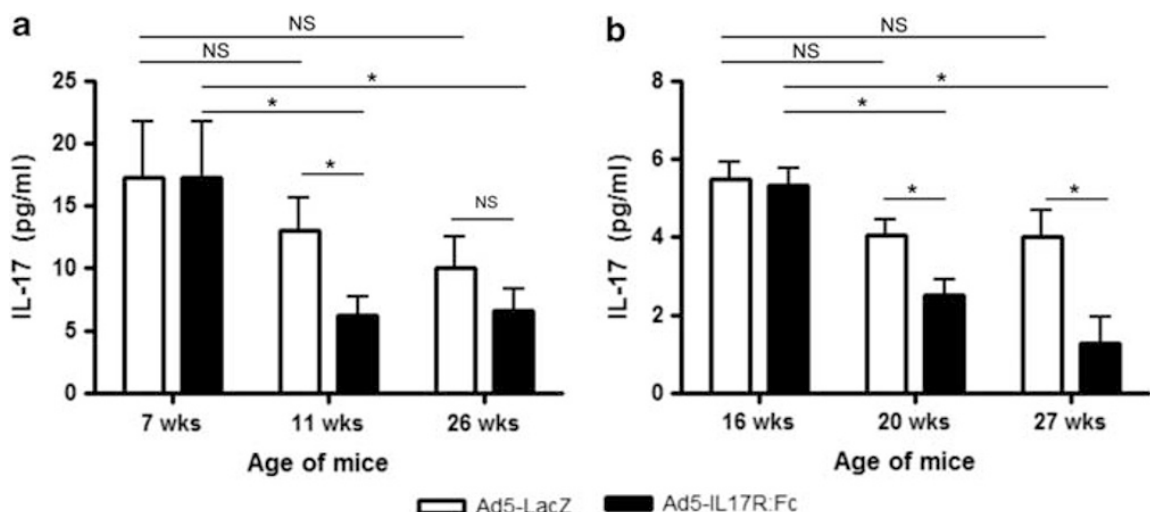

Figure 1 Serum IL17 cytokine levels. Sera were collected at 7 (baseline), 11, and 26 weeks with $n=3$ for each age group (a), and 16 (baseline), 20,27 weeks with $n=3$ for each age group (b). (NS: not significant, $P={ }^{\star}<0.05$ ).

exhibited a marked temporal decrease in IL17 levels at both 3 and 18 weeks post-treatment compared with baseline levels at 7 weeks of age. Mice receiving the control Ad5-LacZ vector showed highest IL17 levels at 7 weeks of age (pre-diseased state), which gradually decreased with age; however, these decreases were not statistically significant and appeared to be consistent with normal progression of the mice. ${ }^{8}$ Similar observations were seen in C57BL/6.NOD-Aec1Aec2 mice treated with Ad5-IL17R:Fc or Ad5-LacZ vector at 17 weeks of age (Figure 1b). Note, however, that by 17 weeks of age, serum IL17 levels were markedly reduced in untreated mice. Nevertheless, the Ad5-IL17:Fc vector, but not the Ad5-LacZ vector, was capable of reducing the serum levels even further. These results support the functional efficacy of the Ad5-IL17:Fc viral vector in suppressing IL17 levels both transiently and stably (up to 18 weeks).

\section{Decreased Numbers of IL17-Producing CD4 + T Cells in the Spleens of Ad5-IL17R:Fc-Transduced Mice}

Mice treated with Ad5-IL17R:Fc or Ad5-LacZ at either 8 or 17 weeks of age were euthanized at 27 and 29 weeks of age, respectively. The splenocytes were isolated and examined for the number of IL17-secreting CD4 $+\mathrm{T}$ cells. As presented in Figure $2 \mathrm{a}$ and $\mathrm{b}$, spleens of C57BL/6.NOD-Aec1Aec2 mice contained about $4 \%$ of CD $4+$ IL17 + T cells at 7 weeks of age and this increased to over $8 \%$ by 19 weeks post-Ad5-LacZ treatment. This result is consistent with the natural aging of the mice (unpublished data) and is not considered a direct effect of treatment with Ad5-LacZ vector. In contrast, as shown in Figure 2c, mice treated with Ad5-IL17R:Fc at 8 weeks of age showed no increased levels of CD4 + IL17 + T cells at 19 weeks post-treatment. A similar functional efficacy of Ad5-IL17R:Fc vector treatment was observed in mice treated at 17 weeks of age (Figure $2 \mathrm{~d}-\mathrm{f}$ ) in which more than a twofold decrease in the number of IL17-secreting CD4 + T cells was seen when examined at 29 weeks of age. In contrast, only a slight decrease in the number of CD $4+$ IL17 + T cells was observed in mice treated with the Ad5-LacZ vector. These data suggest that even though the Ad5 vector is transient and presumably restricted locally to the salivary glands, the effect on C57BL/6.NOD-Aec1Aec2 mice can be systematic and sustained longer than anticipated as evidenced by the decrease in the levels of IL17-secreting cells at 12 or 19 weeks post-treatment.

\section{Reduced SS-like Disease in the Salivary Glands of C57BL/6.NOD-Aec1Aec2 Mice After Transduction with Ad5-IL17R:Fc Vector}

The disease profile in C57BL/6.NOD-Aec1Aec2 mice is well characterized in that they exhibit loss of saliva secretion concomitantly with the appearance of LF in the exocrine glands and increased levels of ANAs. ${ }^{1}$ Thus, to determine the effect of blocking IL17 on the development of SS, mice treated by salivary gland cannulation with either Ad5-IL17R:Fc-blocking vector or Ad5-LacZ control vector at either 8 or 17 weeks of age, were examined for their SS-like disease phenotype at 18 and 12 weeks after cannulation, respectively. Histological examinations of the salivary glands from mice treated with Ad5-IL17R:Fc vector at either 8 or 17 weeks of age revealed a marked decrease in the number of LF at time of euthanization, that is 26 and 29 weeks of age, respectively (Figure 3 ). As presented in Table 1, Figure $3 \mathrm{a}$ and d, of the C57BL/6.NOD-Aec1Aec2 mice whose salivary glands were treated with Ad5-IL17R:Fc vector at 8 weeks of age (early phase treatment), 67\% (6 of 9) had no detectable LF in the salivary glands, whereas $83 \%$ ( 5 of 6 ) mice whose salivary glands were treated with the control Ad5-LacZ vector showed a dramatic increase in the number of LF. Similarly, of the C57BL/6.NOD-Aec1Aec2 mice treated with Ad5-IL17R:Fc vector at 18 weeks of age (late phase treatment), $83 \%$ (10 of 12) had no detectable LF in the salivary glands, compared with $83 \%$ (5 of 6) mice treated with the Ad5-LacZ control vector (Table 1; Figure $3 \mathrm{~g}$ and $\mathrm{j}$ ). Immunofluorescent staining revealed both B and T lymphocytes with IL17 positive cells within the salivary gland ductal and acinar cells and LF of Ad5-LacZ-treated mice (Figure 3b, c, h and i), but not in Ad5-IL17R:Fc-treated mice (Figure 3e and k). 
a
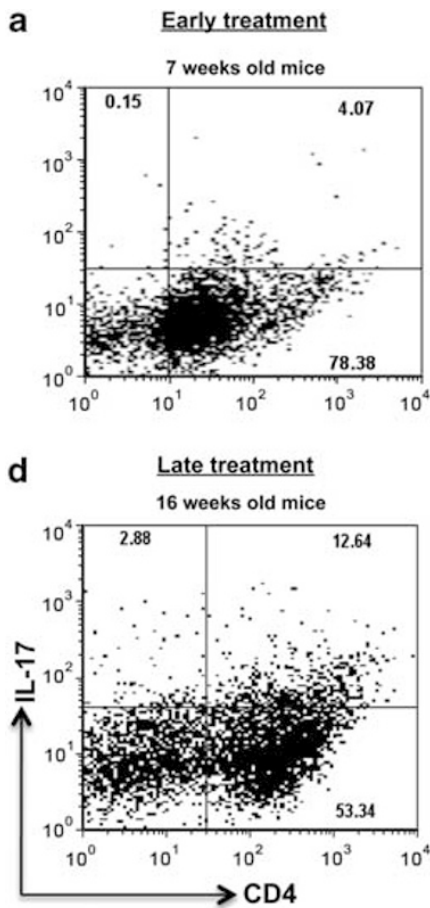

b
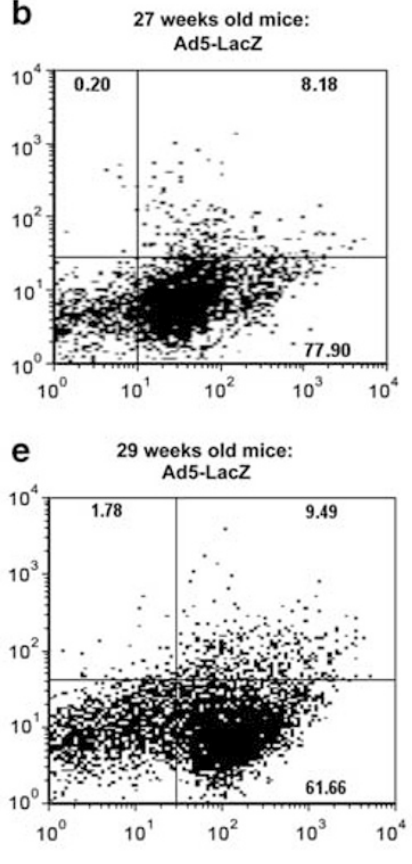

c

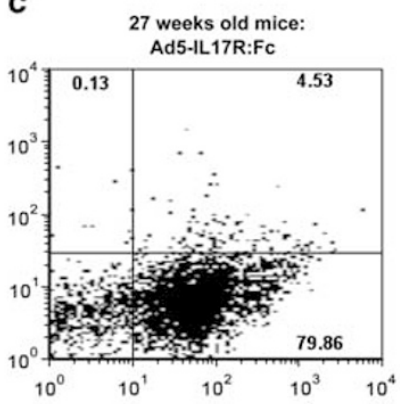

f 29 weeks old mice:

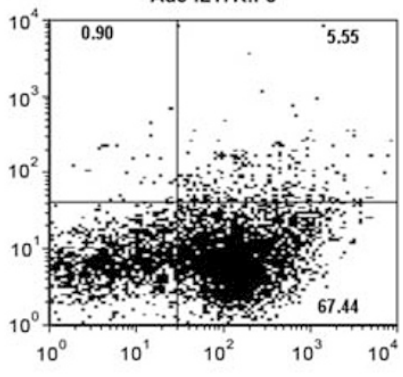

Figure 2 Number of splenic IL17 + CD4 + cells. Spleen cells at 7 weeks old mice (1 week before vector treatment), 27 weeks old mice (19 weeks post-vector treatment) (early treatment, a-c), 16 weeks old mice (1 week before vector treatment), and 29 weeks old mice (12 weeks post-vector treatment) (late treatment, $\mathbf{d}-\mathbf{f}$ ). The data shown are representative of three independent experiments with $n=2$ at each experiment.

Production of autoantibodies, including ANA, may be independent of the $\mathrm{T}_{\mathrm{H}} 17-\mathrm{IL} 17$ system, but it is one of the important criteria in diagnosing SS disease. Sera obtained from mice treated with the Ad5-LacZ vector at 8 weeks of age in the early treatment group exhibited the expected changes in ANA-staining pattern, evolving from a faint cytoplasmic/ nuclear to a homogenous nuclear profile between 7 and 26 weeks of age, indicative of an SS disease ANA-staining profile (Figure $4 \mathrm{a}$ and $\mathrm{b}$ ). In contrast, mice that received Ad5-IL17R:Fc vector maintained weak cytoplasmic/nuclearstaining pattern between 7 and 26 weeks of age, or 18 weeks post-treatment (Figure 4c). As anticipated, sera collected from 16 weeks old C57BL/6.NOD-Aec1Aec2 mice, that is 1 week before cannulation in the late treatment group, were positive for ANA with a homogenous nuclear pattern. The ANA profile remained homogenous nuclear with higher intensity at 10 weeks post-treatment with Ad5-LacZ vector. Paradoxically, mice treated with Ad5-IL17R:Fc vector shifted from homogenous nuclear to normal cytoplasmic/nuclear ANA profile (Figure 4f). Therefore, blocking IL17 appears to have a significant effect on the ANA profiles in $\mathrm{SS}^{\mathrm{S}} \mathrm{C} 57 \mathrm{BL} /$ 6.NOD-Aec1Aec2 mice.

\section{Preventing Secretory Dysfunction or Restoring Normal Saliva Flow in C57BL/6.NOD-Aec1Aec2 Mice After Ad5-IL17R:Fc Treatment}

To determine whether the Ad5-IL17R:Fc vector is capable of preventing SS disease progression in C57BL/6.NOD-
Aec1Aec2 mice when treated before development of SS (ie 7 weeks of age), or restoring normal saliva secretion when treated at the late stage of SS development (ie 17 weeks of age), saliva volumes were collected and measured post-cannulation. As presented in Figure 5a, mice whose salivary glands were cannulated at 7 weeks of age with Ad5-IL17R:Fc vector retained normal saliva flow at 3 and 18 weeks postcannulation compared with baseline level at 7 weeks of age. In contrast, mice that received control Ad5-LacZ vector showed the expected gradual decrease in saliva secretion over this same time intervals. Similarly, mice whose salivary glands were treated with Ad5-IL17R:Fc vector at 17 weeks of age exhibited temporally progressive increases in saliva secretion over the 11 weeks follow-up period (Figure 5b), suggesting a significant recovery of salivary function. This result indicates that blocking IL17 is capable of preventing development of SS when carried out before onset of disease, and even restoring normal salivary function when carried out at a later stage of the disease.

\section{DISCUSSION}

The $\mathrm{T}_{\mathrm{H}}$ 17-derived IL17 (IL17A) cytokine is a potent inflammatory cytokine that has been implicated in a growing list of autoimmune diseases, for example multiple sclerosis, Crohn's disease, rheumatoid arthritis, psoriasis, systemic lupus erythematosus, and SS, as well as autoimmunity in animal models. ${ }^{3}$ The consequence of $\mathrm{T}_{\mathrm{H}}$ 17/IL17 activation includes, in addition to the production, the IL17 family of 

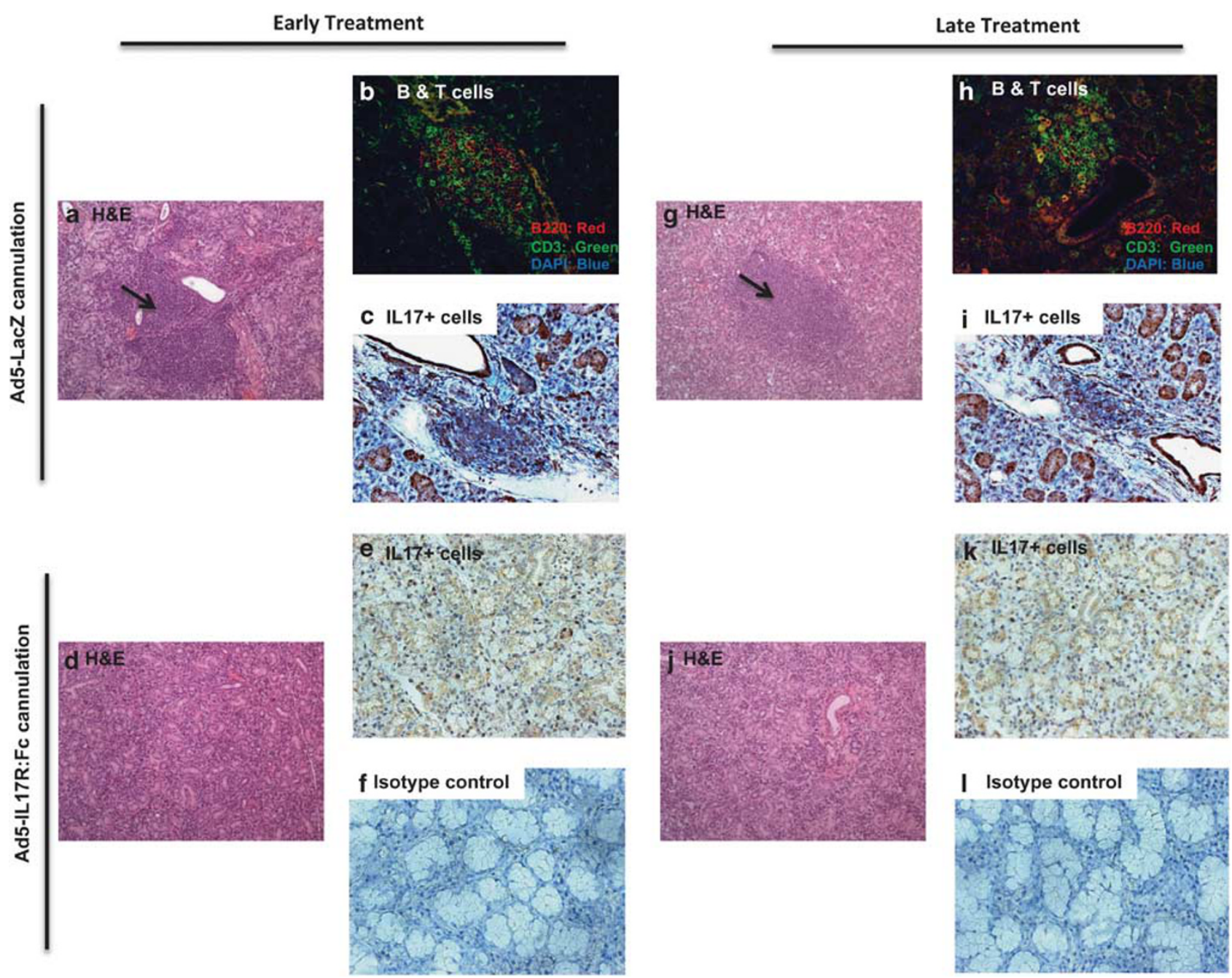

Figure 3 Histological analyses of salivary glands. Examination of the salivary glands in mice cannulated at 8 weeks or indicated 'early treatment' $(n=15)(\mathbf{a}-\mathbf{f})$ or 17 weeks of age or indicated 'late treatment' $(n=18)(\mathbf{g}-\mathbf{I})$ with either Ad5-LacZ or Ad5-IL17R:Fc vectors at $10^{7}$ viral particles per gland. Black arrows indicate representative lymphocytic infiltrate in H\&E sections $(\mathbf{a}, \mathbf{d}, \mathbf{g}$, j), immunofluorescent staining for CD3 $+\mathrm{T}$ and B220 + B cells $(\mathbf{b}$, $\mathbf{h}$ ), and immunohistochemical staining for IL17 cells (c, e, i, k). Isotype control for IL17 antibody was performed with rabbit IgG (f, I). Images were taken at $\times 200$ magnification at constant exposure of $0.3 \mathrm{~s}$ using Zeiss Axiovert $200 \mathrm{M}$ microscope (Carl Zeiss).

Table 1 Quantification of lymphocytic foci (LF) in salivary glands

\begin{tabular}{lccccccc}
\hline & \multicolumn{3}{c}{ Ad5-LacZ } & & \multicolumn{3}{c}{ Ad5-IL17R:Fc } \\
\cline { 2 - 3 } \cline { 6 - 8 } & No LF & LF & Mean LF & No LF & LF & Mean LF \\
\hline Early*** & $1^{\text {a }}(17 \%)^{\mathrm{b}}$ & $5(83 \%)$ & $3.6 \pm 0.6^{\mathrm{c}}$ & $6(67 \%)$ & $3(33 \%)$ & $1 \pm 0.0$ \\
Late $^{* * *}$ & $1(17 \%)$ & $5(83 \%)$ & $3.6 \pm 0.9$ & & $10(83 \%)$ & $2(17 \%)$ & $1 \pm 0.0$
\end{tabular}

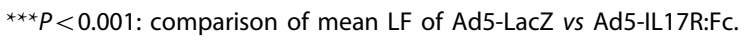

${ }^{\mathrm{a}}$ Number of mice.

bercentage of mice.

${ }^{c}$ Mean number of $L F \pm S E M$ per histological salivary gland section.

cytokines, the production of IL21, IL22, chemokines (MIP-2, CXCL1, CXCL2, CXCL5), and matrix metalloproteases (MMP3 and MMP13), ${ }^{16}$ all actively involved in tissue inflammation. Interaction of the IL17 with its receptors evokes activation of CXCL8, resulting in recruitment of neutrophils to the site of injury. Thus, IL17 has emerged as an ideal therapeutic target for autoimmune disease. In this study, we sought to examine the effect(s) of inhibiting IL17 on SS development using an adenoviral vector in a mouse model of SS. The results suggest that inhibiting IL17 at early disease stage can prevent the onset of SS development, specifically the absence of lymphocytic infiltration in the salivary glands, retention of normal ANA profiles, and no loss in saliva secretion. Likewise, inhibiting IL17 at a later disease stage could rescue salivary gland function by ameliorating lymphocytic infiltrations, normalizing ANA profiles, and more importantly recovering saliva secretion.

The design of this study has taken advantage of several important observations: (1) the temporal disease profile of SS ${ }^{S}$ C57BL/6.NOD-Aec1Aec2 mice is well defined at both 


\section{Early Treatment (cannulated at 8 wks old)}

a 7 wks old B6.NOD-Aec1Aec2

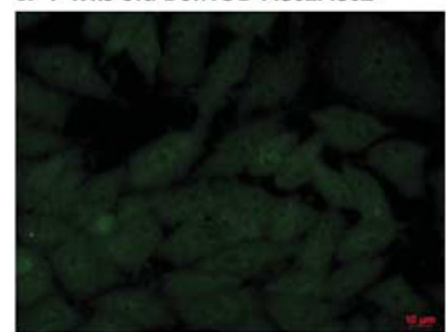

b 26 wks old Ad5-LacZ treated

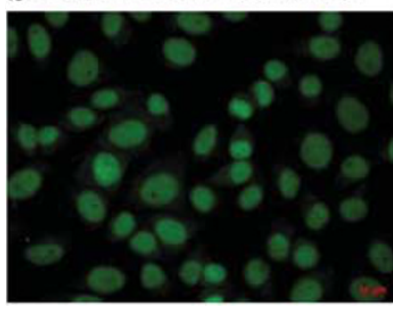

C 26 wks old Ad5-IL17R:Fc treated

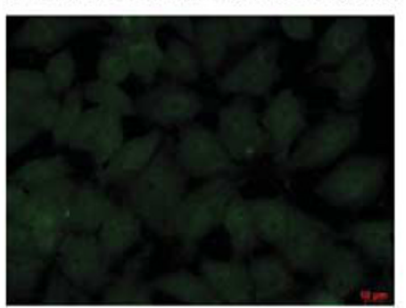

Late Treatment (cannulated at 17 wks old)
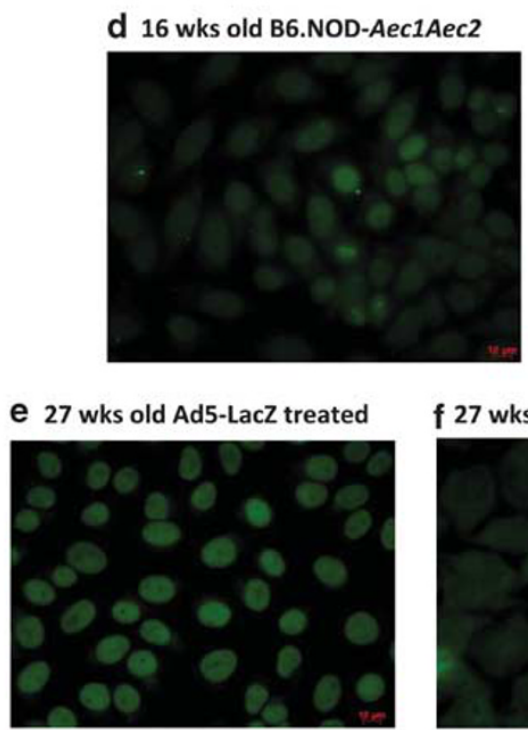

f 27 wks old Ad5-IL17R:Fc treated

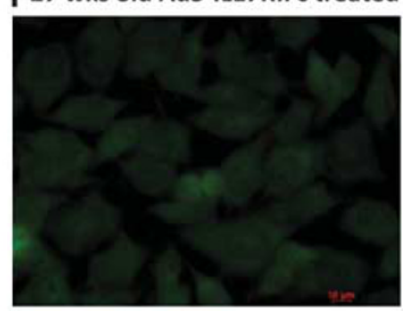

Figure 4 Identification of ANA. Representative patterns of cellular staining of HEp2 cells by sera diluted 1/40 prepared from mice cannulated with Ad5-LacZ or Ad5-IL17R:Fc vectors at 8 weeks of age (early treatment) (a-c), and 17 weeks of age (late treatment) (d-f). Sera were collected 1 week before cannulation $(\mathbf{a}, \mathbf{d})$ and at indicated times of euthanization $(\mathbf{b}, \mathbf{c}, \mathbf{e}, \mathbf{f})$ for both vector-treated groups. ANA in sera was tested using HEp2 cells substrate. Representative patterns were determined with $n=3$ for each time point presented.
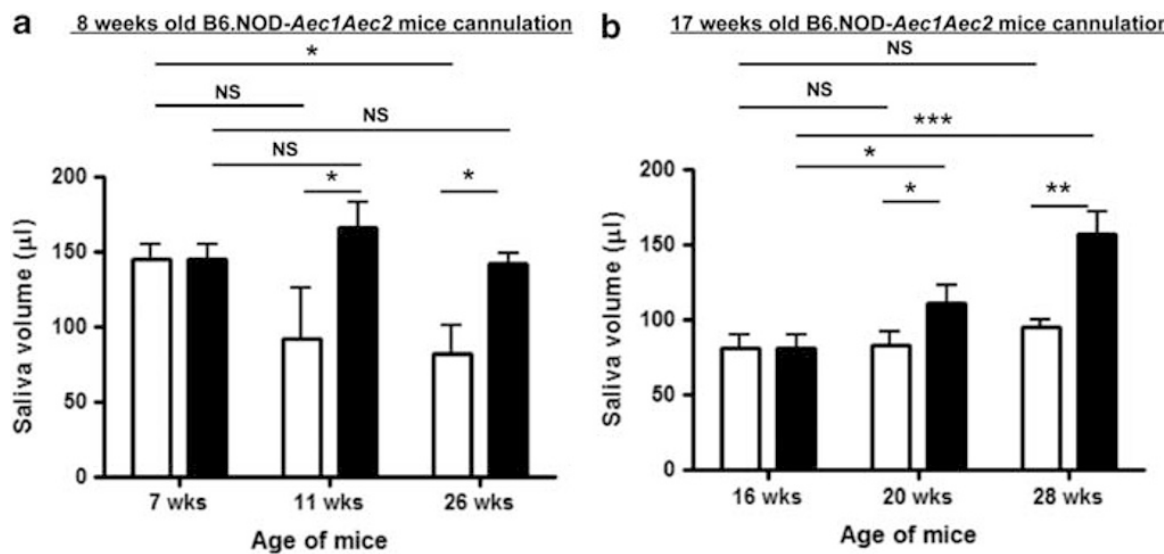

Figure 5 Secretory function of salivary glands. Saliva collected at 7 or 1 week before cannulation (baseline), 11 and 26 weeks of age (Ad5-LacZ, $n=6$ and Ad5-IL17R:Fc, $n=8$ ) (a), and 16 or 1 week before cannulation, 20 and 28 weeks of age (Ad5-LacZ, $n=9$ and Ad5-IL17R:Fc, $n=10)$ (b). Statistical analysis was used to determine the significance between the Ad5-LacZ- and Ad5-IL17R:Fc-treated mice at each time point. (NS, not significant, $\left.P={ }^{*}<0.05, P={ }^{* *}<0.01, P={ }^{* *}<0.001\right)$.

the genetic and pathological levels, ${ }^{1,2}$ (2) histological examinations of salivary gland biopsies from both SS patients and C57BL/6.NOD-Aec1Aec2 mice indicate the presence of the IL23/ $\mathrm{T}_{\mathrm{H}} 17 / \mathrm{IL} 17$ system within LF, whereas plasma IL17 levels in SS patients correlate with the disease state, ${ }^{8}$ and (3) retrograde cannulation of the salivary glands in mice through the submandibular ducts can be used to deliver viral vectors encoding recombinant proteins. $^{27-29}$ Cannulations were carried out at two different ages corresponding to time points of expected early-stage (6-8 weeks of age) and later-stage (15-17 weeks of age) pathogenesis. The later-stage studies were carried out based on the fact that C57BL/6.NODAec1Aec2 mice still have intact glands and partial salivary flow rates. With this design, we have been able to examine the direct effect of IL17 blockage as a therapeutic target in preventing either development or onset of SS. A possible weakness in the present design to be considered is the use of the Ad5-based vector system known to express the recombinant protein for a relatively shorter defined time span, ${ }^{32,33}$ and, therefore, possibly only transient immunological functions. Interestingly, as presented in this study, the effect of the Ad5 vector using IL17R:Fc was quite 
stable up to 19 weeks post-treatment, possibly contributed by the stability of the receptor portion, which can be enhanced and prolonged because of the Fc protein fusion. Numerous studies have shown that $\mathrm{Fc}$ fusion proteins extend the serum half-life of the partner protein, limit renal clearance, and significantly promote protein secretion with high expression. ${ }^{34}$ Furthermore, even though adenoviral vectors are capable of inducing immunological responses, ${ }^{35,36}$ the lowdosage treatment ( $10^{7}$ viral particles per salivary gland) used in this study was well tolerated and do not elicit any observable side effects.

In addition to the longer duration or persistence of the Ad5 vector in the cannulated mice, the effect of the vectors appears to be systemic, as defined by changes observed in both sera and spleens of the Ad5-IL17R:Fc-treated mice. Even though SS targets primarily the exocrine glands, specifically the salivary and lacrimal glands, the pathology can be systemic thereby affecting multiple organs. Extensive studies by Bruce Baum's laboratory have provided significant evidence into the systemic effect of the adenovirus transduction. ${ }^{37-40}$ As shown by Adesanya et $a l l^{31}$ retrograde salivary gland cannulation at high vector dose can injure acinar cells, which likely compromise the integrity of the mucosal barrier allowing for leakage of the vector systemically. Further studies by Kagami et $a l^{39}$ and He $e t a l^{41}$ provide evidence that ductal cannulation of salivary glands can result in systemic effects because of the secretory nature of the salivary glands, which are well endowed with protein synthesis organelles and secretory machinery. As observed in this study, the systemic spread of the vector is quite expected and promising

Our studies have indicated that generation of LF in the salivary glands ${ }^{1}$ requires an intricate and synchronized action between $\mathrm{T}_{\mathrm{H}} 1, \mathrm{~T}_{\mathrm{H}} 2$, and $\mathrm{T}_{\mathrm{H}} 17$ cells. Study by Jonsson et al ${ }^{42}$ has indicated that some LF form germinal center-like structures and that the appearance of such structures correlate with a more severe disease and higher production of autoantibodies in human patients. We have shown that the initial infiltrating cells are $T_{H} 1$ cells producing IFN- $\gamma$, which directly mediates the up-regulation of adhesion molecules, consequently recruiting inflammatory cells such as $\mathrm{T}_{\mathrm{H}} 2$ and $\mathrm{T}_{\mathrm{H}} 17$ cells to the glands. The destruction of the glands is suggested to be executed by the pathogenic potential of IL17 cytokine (Nguyen et al, unpublished data). More importantly, a recent study $^{24}$ has found that IL17 is needed to maintain the structure and formation of GC-like organization in an autoimmune animal model; therefore, blocking it with Ad517R:Fc vector has been shown to destroy the integrity of the GC by the dissociation of $\mathrm{B}$ cell from $\mathrm{CD} 4+\mathrm{T}$ cells within the follicles. Furthermore, Doreau et $a l^{43}$ have shown that IL17 alone or in combination with BAFF (B cells activating factor) can influence the survival, proliferation, and differentiation of B lymphocytes and maintain the existence of self-reactive B cells. These seminal studies clearly support our findings in which blocking the activity of IL17 prevented the generation of LF in the glands or dissociate the existing LF because of the lack of survival or maintenance signals produced by IL17, and this dissipation of the LF ameliorates the formation of self-reactive $\mathrm{B}$ cell, thereby eliminating the emergence of autoreactive antibodies.

In conclusion, reduction of IL17A levels by Ad5-IL17R:Fcblocking vectors suppresses features of $\mathrm{SS}$ in $\mathrm{SS}^{\mathrm{S}}$ mice, demonstrating the major function this cytokine has in the development of this autoimmune disease. How this one cytokine affects the various features of autoimmunity, and at what level or time point, will require additional studies. Nevertheless, the simple and relatively safe cannulation procedure to introduce the Ad5-IL17R:Fc vector directly into the targeted glands suggests that this intervention therapy should be more thoroughly investigated. The promising aspect of the present studies is that intervention at late stage of SS can provide protection from further destruction or recovery of salivary gland function. Longer observation is needed to determine the long-term effect of adenoviral vectors per se and IL17 at late-stage disease. The future application of adeno-associated viral vectors, which provide a more stable and persistent factor expression, could advance gene therapy application to future treatment of SS.

\section{ACKNOWLEDGEMENTS}

We thank Dr Jay K Kolls and Dr Julie Bindas (Children's Hospital of Pittsburgh) for generously providing the Ad5-LacZ and Ad5-IL17R:FC vectors, and Dr Phil Cohen for his critical reading of the manuscript and helpful suggestions. We greatly appreciate the assistance of Dr Craig Meyers and Dr Nicholas Muzyczka for the use of the microscope. This work was supported by PHS Grant K99 DE018958 grant (CQN) from the NIDCR. ABP and CQN were supported by PHS Grant 1R21AI081952 from NIAID. HY and JAC were supported by an NIH, NIDCR intramural research grant.

\section{DISCLOSURE/CONFLICT OF INTEREST}

The authors declare no conflict of interest.

1. Nguyen $C Q, C h a S R$, Peck $A B$. Sjögren's syndrome (SjS)-like disease of mice: the importance of $B$ lymphocytes and autoantibodies. Front Biosci 2007;12:1767-1789.

2. Nguyen $C Q$, Peck $A B$. Unraveling the pathophysiology of Sjogren syndrome-associated dry eye disease. Ocul Surf 2009;7:11-27.

3. Lee BH, Tudares MA, Nguyen CQ. Sjogren's syndrome: an old tale with a new twist. Arch Immunol Ther Exp (Warsz) 2009;57:57-66.

4. Nguyen KH, Brayer J, Cha S, et al. Evidence for antimuscarinic acetylcholine receptor antibody-mediated secretory dysfunction in nod mice. Arthritis Rheum 2000;43:2297-2306.

5. Nguyen $\mathrm{CQ}, \mathrm{Gao} \mathrm{JH}$, Kim H, et al. IL-4-STAT6 signal transductiondependent induction of the clinical phase of Sjogren's syndrome-like disease of the nonobese diabetic mouse. J Immunol 2007;179: 382-390.

6. Nguyen C, Cornelius J, Singson E, et al. Role of complement and B lymphocytes in Sjogren's syndrome-like autoimmune exocrinopathy of NOD.B10-H2b-mice. Mol Immunol 2006;43:1332-1339.

7. Nguyen CQ, Kim H, Cornelius JG, et al. Development of Sjogren's syndrome in NOD-derived autommune-prone C57BL/6.NOD-Aec1Aec2 mice is dependent on complement component-3. J Immunol 2007;179:2318-2329.

8. Nguyen $\mathrm{CQ}, \mathrm{Hu} \mathrm{MH}, \mathrm{Li}$ Y, et al. Salivary gland tissue expression of interleukin-23 and interleukin-17 in Sjogren's syndrome: findings in humans and mice. Arthritis Rheum 2008;58:734-743.

9. Sakai A, Sugawara Y, Kuroishi T, et al. Identification of IL-18 and Th17 cells in salivary glands of patients with Sjogren's syndrome, and 
amplification of IL-17-mediated secretion of inflammatory cytokines from salivary gland cells by IL-18. J Immunol 2008;181:2898-2906.

10. Harrington LE, Hatton RD, Mangan PR, et al. Interleukin 17-producing CD4+ effector $T$ cells develop via a lineage distinct from the $T$ helper type 1 and 2 lineages. Nat Immunol 2005;6:1123-1132.

11. Park $H, L i Z$, Yang XO, et al. A distinct lineage of CD4 T cells regulates tissue inflammation by producing interleukin 17. Nat Immunol 2005;6:1133-1141.

12. Veldhoen $M$, Hocking RJ, Atkins $C J$, et al. TGFbeta in the context of an inflammatory cytokine milieu supports de novo differentiation of IL-17producing T cells. Immunity 2006;24:179-189.

13. Bettelli E, Carrier Y, Gao W, et al. Reciprocal developmental pathways for the generation of pathogenic effector TH17 and regulatory T cells. Nature 2006;441:235-238.

14. Mangan PR, Harrington LE, O'Quinn DB, et al. Transforming growth factor-beta induces development of the $\mathrm{T}(\mathrm{H}) 17$ lineage. Nature 2006;441:231-234.

15. Ivanov II, McKenzie BS, Zhou L, et al. The orphan nuclear receptor RORgammat directs the differentiation program of proinflammatory IL-17+ T helper cells. Cell 2006;126:1121-1133.

16. Weaver CT, Hatton RD, Mangan PR, et al. IL-17 family cytokines and the expanding diversity of effector $\mathrm{T}$ cell lineages. Annu Rev Immunol 2007;25:821-852.

17. Kastelein RA, Hunter CA, Cua DJ. Discovery and biology of IL-23 and IL27: related but functionally distinct regulators of inflammation. Annu Rev Immunol 2007;25:221-242.

18. Zhou L, Lopes JE, Chong MM, et al. TGF-beta-induced Foxp3 inhibits $\mathrm{T}(\mathrm{H}) 17$ cell differentiation by antagonizing RORgammat function. Nature 2008;453:236-240.

19. Duerr RH, Taylor KD, Brant SR, et al. A genome-wide association study identifies IL23R as an inflammatory bowel disease gene. Science 2006;314:1461-1463.

20. Hue $\mathrm{S}$, Ahern $\mathrm{P}$, Buonocore $\mathrm{S}$, et al. Interleukin-23 drives innate and $\mathrm{T}$ cell-mediated intestinal inflammation. J Exp Med 2006;203:2473-2483.

21. Cua DJ, Sherlock J, Chen Y, et al. Interleukin-23 rather than interleukin12 is the critical cytokine for autoimmune inflammation of the brain. Nature 2003:421:744-748.

22. Ye $\mathrm{P}$, Rodriguez $\mathrm{FH}$, Kanaly $\mathrm{S}$, et al. Requirement of interleukin 17 receptor signaling for lung CXC chemokine and granulocyte colonystimulating factor expression, neutrophil recruitment, and host defense. J Exp Med 2001;194:519-527.

23. Schwarzenberger $\mathrm{P}$, La Russa V, Miller $\mathrm{A}$, et al. IL-17 stimulates granulopoiesis in mice: use of an alternate, novel gene therapyderived method for in vivo evaluation of cytokines. J Immunol 1998;161:6383-6389.

24. Hsu HC, Yang $\mathrm{P}$, Wang J, et al. Interleukin 17-producing $\mathrm{T}$ helper cells and interleukin 17 orchestrate autoreactive germinal center development in autoimmune BXD2 mice. Nat Immunol 2008;9: 166-175.

25. Kolls J, Peppel K, Silva M, et al. Prolonged and effective blockade of tumor necrosis factor activity through adenovirus-mediated gene transfer. Proc Natl Acad Sci USA 1994;91:215-219.

26. Zheng C, Baum BJ. Evaluation of promoters for use in tissue-specific gene delivery. Methods Mol Biol 2008;434:205-219.
27. Kok MR, Yamano $S$, Lodde $B M$, et al. Local adeno-associated virusmediated interleukin 10 gene transfer has disease-modifying effects in a murine model of Sjogren's syndrome. Hum Gene Ther 2003;14: $1605-1618$.

28. Kok MR, Voutetakis A, Yamano S, et al. Immune responses following salivary gland administration of recombinant adeno-associated virus serotype 2 vectors. J Gene Med 2005;7:432-441.

29. Lodde BM, Mineshiba F, Wang J, et al. Effect of human vasoactive intestinal peptide gene transfer in a murine model of Sjogren's syndrome. Ann Rheum Dis 2006;65:195-200.

30. Shai E, Falk $H$, Honigman A, et al. Gene transfer mediated by different viral vectors following direct cannulation of mouse submandibular salivary glands. Eur J Oral Sci 2002;110:254-260.

31. Adesanya MR, Redman RS, Baum BJ, et al. Immediate inflammatory responses to adenovirus-mediated gene transfer in rat salivary glands. Hum Gene Ther 1996;7:1085-1093.

32. Delporte C, Redman RS, Baum BJ. Relationship between the cellular distribution of the alpha(v)beta3/5 integrins and adenoviral infection in salivary glands. Lab Invest 1997;77:167-173.

33. Cotrim AP, Mineshiba F, Sugito $T$, et al. Salivary gland gene therapy. Dent Clin North Am 2006;50:157-173, vii.

34. Huang C. Receptor-Fc fusion therapeutics, traps, and MIMETIBODY technology. Curr Opin Biotechnol 2009;20:692-699.

35. Crystal RG, Harvey BG, Wisnivesky JP, et al. Analysis of risk factors for local delivery of low- and intermediate-dose adenovirus gene transfer vectors to individuals with a spectrum of comorbid conditions. Hum Gene Ther 2002;13:65-100.

36. Harvey BG, Maroni J, O'Donoghue KA, et al. Safety of local delivery of low- and intermediate-dose adenovirus gene transfer vectors to individuals with a spectrum of morbid conditions. Hum Gene Ther 2002;13:15-63.

37. Zheng C, Voutetakis A, Kok MR, et al. Toxicity and biodistribution of a first-generation recombinant adenoviral vector, in the presence of hydroxychloroquine, following retroductal delivery to a single rat submandibular gland. Oral Dis 2006;12:137-144.

38. O'Connell BC, Zheng C, Jacobson-Kram D, et al. Distribution and toxicity resulting from adenoviral vector administration to a single salivary gland in adult rats. J Oral Pathol Med 2003;32:414-421.

39. Kagami H, O'Connell BC, Baum BJ. Evidence for the systemic delivery of a transgene product from salivary glands. Hum Gene Ther 1996;7:2177-2184

40. Wang J, Voutetakis A, Mineshiba F, et al. Effect of serotype 5 adenoviral and serotype 2 adeno- associated viral vector-mediated gene transfer to salivary glands on the composition of saliva. Hum Gene Ther 2006;17:455-463

41. He X, Goldsmith CM, Marmary $Y$, et al. Systemic action of human growth hormone following adenovirus-mediated gene transfer to rat submandibular glands. Gene Ther 1998;5:537-541.

42. Jonsson MV, Skarstein K, Jonsson R, et al. Serological implications of germinal center-like structures in primary Sjogren's syndrome. J Rheumatol 2007;34:2044-2049.

43. Doreau A, Belot A, Bastid J, et al. Interleukin 17 acts in synergy with B cellactivating factor to influence $B$ cell biology and the pathophysiology of systemic lupus erythematosus. Nat Immunol 2009;10:778-785. 